\title{
High-Grade T1 on Re-Transurethral Resection after Initial High-Grade T1 Confers Worse Oncological Outcomes: Results of a Multi-Institutional Study
}

\author{
Matteo Ferro $^{\mathrm{a}}$ Mihai Dorin Vartolomei ${ }^{\mathrm{a}-\mathrm{c}}$ Francesco Cantiello $^{\mathrm{d}}$ \\ Giuseppe Lucarelli $^{\text {e Savino M. Di Stasi }}{ }^{f}$ Rodolfo Hurle ${ }^{g}$ Giorgio Guazzonig
}

Gian Maria Busetto $^{\text {h }}$ Ettore De Berardinis ${ }^{h}$ Rocco Damiano ${ }^{d}$ Sisto Perdonài

Marco Borghesi ${ }^{j}$ Riccardo Schiavina ${ }^{j} \quad$ Gilberto L. Almeidak ${ }^{k i e r l u i g i ~ B o v e l}$

Estevao Lima ${ }^{m}$ Giovanni Grimaldi ${ }^{m}$ Riccardo Autorino ${ }^{n}$ Nicolae Crisan ${ }^{\circ}$

\author{
Abdal Rahman Abu Farhan ${ }^{d}$ Paolo Verze $^{p} \quad$ Michele Battaglia $^{e}$ \\ Vincenzo Serretta ${ }^{q}$ Giorgio Ivan Russo ${ }^{r}$ Giuseppe Morgiar Gennaro Musia

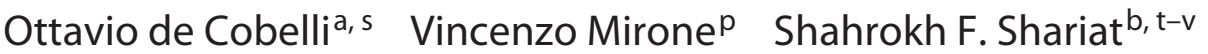

\begin{abstract}
${ }^{a}$ Division of Urology, European Institute of Oncology, Milan, Italy; ${ }^{b}$ Department of Urology, Medical University of Vienna, Vienna, Austria; ' Department of Cell and Molecular Biology, University of Medicine and Pharmacy, Tirgu Mures, Romania; ${ }^{\mathrm{d} D e p a r t m e n t}$ of Urology, Magna Graecia University of Catanzaro, Catanzaro, Italy; ${ }^{\mathrm{e}}$ Department of Emergency and Organ Transplantation, Urology, Andrology and Kidney Transplantation Unit, University of Bari, Bari, Italy; ${ }^{f}$ Department of Experimental Medicine and Surgery, Tor Vegata University, Rome, Italy; ${ }^{9}$ Department of Urology, Istituto Clinico Humanitas IRCCS, Clinical and Research Hospital, Humanitas University, Department

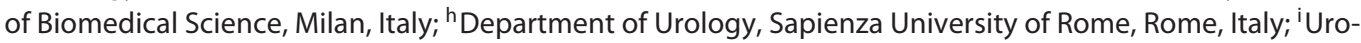
Gynecological Department, Istituto Nazionale per lo Studio e la Cura dei Tumori, Fondazione "G. Pascale"-IRCCS, Naples, Italy; ${ }^{j}$ Department of Urology, University of Bologna, Bologna, Italy; ${ }^{k}$ Departamento de Urologia, University of Vale do Itajai, Itajai, Brazil; 'UOC of Urology Department of Experimental Medicine and Surgery, Tor Vergata University of Rome, Rome, Italy; ${ }^{\mathrm{m}}$ Life and Health Sciences Research Institute, University of Minho, Braga, Portugal; ${ }^{n}$ Division of Urology, Virginia Commonwealth University, Richmond, VA, USA; ${ }^{\circ}$ Department of Urology, University of Medicine and Pharmacy "Iuliu Hațeganu", Cluj-Napoca, Romania; ${ }^{P}$ Department of Neurosciences, Sciences of Reproduction and Odontostomatology, Urology Unit, University of Naples "Federico II", Naples, Italy; "Department of Surgical, Oncological and Stomatological Sciences, University of Palermo, Palermo, Italy; ' Department of Surgery, Urology Section, University of Catania, Catania, Italy; ${ }^{5}$ Università degli Studi di Milano, Via Festa del Perdono 7, Milan, Italy; ${ }^{\mathrm{t}}$ Karl Landsteiner Institute of Urology and Andrology, Vienna, Austria; ${ }^{\mathrm{u} D e p a r t m e n t}$ of Urology, University of Texas Southwestern Medical Center, Dallas, TX, USA; ${ }^{\vee}$ Department of Urology, Weill Cornell Medical College, New York, NY, USA
\end{abstract}

\section{Keywords}

High-grade · Bladder cancer · High risk · Transurethral resection of bladder tumor . Second look resection

\section{KARGER}

(c) 2018 S. Karger AG, Basel

E-Mail karger@karger.com

www.karger.com/uin
Mihai Dorin Vartolomei

Division of Urology

European Institute of Oncology

Via Ripamonti 435, IT-20141 Milan (Italy)

E-Mail mihai.vartolomei@umftgm.ro 


\section{Abstract}

Introduction: The aim of this multicenter study was to investigate the prognostic impact of residual $\mathrm{T} 1$ high-grade (HG)/G3 tumors at re-transurethral resection (TUR of bladder tumor) in a large multi-institutional cohort of patients with primary T1 HG/G3 bladder cancer (BC). Patients and Methods: The study period was from January 2002 to December 2012. A total of 1,046 patients with primary T1 HG/G3 and who had non-muscle invasive BC (NMIBC) on reTUR followed by adjuvant intravesical Bacillus CalmetteGuerin (BCG) therapy with maintenance were included. Endpoints were time to disease recurrence, progression, and overall and cancer-specific death. Results: A total of 257 (24.6\%) patients had residual T1 HG/G3 tumors. The presence of concomitant carcinoma in situ, multiple and large tumors $(>3 \mathrm{~cm})$ at first TUR were associated with residual T1 $H G / G 3$. Five-year recurrence-free survival (RFS), progression-free survival (PFS), overall survival (OS), and cancerspecific survival (CSS) were $17 \%(\mathrm{Cl} 11.8-23) ; 58.2 \%$ (Cl 50.765); 73.7\% (Cl 66.3-79.7); and 84.5\% (Cl 77.8-89.3), respectively, in patients with residual $\mathrm{T} 1 \mathrm{HG} / \mathrm{G} 3$, compared to 36.7\% (Cl 32.8-40.6); 71.4\% (Cl 67.3-75.2); 89.8\% (Cl 86.692.3); and $95.7 \%$ (Cl 93.4-97.3), respectively, in patients with NMIBC other than T1 HG/G3 or T0 tumors. Residual T1 HG/ G3 was independently associated with RFS, PFS, OS, and CSS in multivariable analyses. Conclusions: Residual T1 HG/ G3 tumor at re-TUR confers worse prognosis in patients with primary $\mathrm{T} 1 \mathrm{HG} / \mathrm{G} 3$ treated with maintenance BCG. Patients with residual $\mathrm{T} 1 \mathrm{HG} / \mathrm{G} 3$ for primary $\mathrm{T} 1 \mathrm{HG} / \mathrm{G} 3$ are very likely to fail BCG therapy alone.

(c) 2018 S. Karger AG, Basel

\section{Introduction}

An estimated 429,800 new cases of bladder cancer (BC) are diagnosed worldwide annually and almost 165,000 patients succumb to it $[1,2]$. Approximately $75 \%$ of these patients, in Western countries have nonmuscle-invasive BC (NMIBC) at diagnosis [3]. Highgrade $\mathrm{T} 1$ (T1 HG/G3) is the NMIBC subtype with the highest risk of disease recurrence and progression [4]. The standard treatment of all T1 HG/G3 considered for bladder preservation is a re-transurethral resection (TUR), if NMIBC or T0 is identified on re-TUR then adjuvant intravesical Bacilus Calmette-Guerin (BCG) therapy with maintenance is often the therapy of choice $[3,5,6]$. However, up to $40 \%$ of patients with initial T1 HG/G3 eventually fail intravesical therapy with BCG within 5 years [7].
Because of this high rate of disease progression to MIBC and the differentially worse prognosis of patients who experience disease progression compared to those presenting with MIBC, there has been intense research to identify T1 HG/G3 patients who are likely to fail BCG therapy and may therefore possibly benefit from intensified therapy such as early radical cystectomy (RC) [8-13]. While, the American Urological Association guidelines suggest to offer an initial RC to patients with persistent T1 HG/G3 on re-TUR, the European Association of Urology (EAU) guidelines does not give specific recommendations [14]. Indeed, the presence of $\mathrm{T} 1$ on re-TUR after the initial TUR showing T1 HG/G3 confers worse survival in singlecenter or multicenter heterogeneous datasets. These studies are impacted by the negative effects of variable and sometimes older BCG therapy schemes among other design limitations $[15,16]$. Persistent disease after initial T1 HG/G3 BC is indeed in approximately 33$55 \%$ of patients [15-17], supporting the recommendation for re-TUR in all patients with T1 HG/G3 [3, 5]. But, in a large multi-institutional cohort of 2451 patients with T1 HG/G3 tumors treated with BCG, a second resection $(n=935)$ improved recurrence-free survival (RFS), progression-free survival (PFS), overall survival (OS), and cancer-specific survival (CSS) only in patients without muscle in the specimen in the initial resection [15].

The aim of this multicenter study was to investigate and validate the prognostic impact of residual T1 HG/G3 tumors at re-TUR in a large multi-institutional cohort of patients who had T1 HG/G3 NMIBC on initial TUR and who were treated with intravesical BCG therapy according to guidelines.

\section{Patients and Methods}

Patient Selection and Data Collection

After an institutional-review-board approval in each institution, with all participating sites providing institutional data sharing agreements prior to the initiation of the study, a total of 1,155 patients with initial G3/HGT1 were treated between January 1, 2002 and December 31, 2012 at 13 academic institutions. The inclusion criteria were re-TUR within 4-6 weeks followed by intravesical BCG therapy with maintenance, generally according to the EAU guidelines at the time. Patients treated with intravesical chemotherapy were excluded (109 patients). Demographical, clinical, pathological, and outcomes data were collected and entered in a computerized database. Data integrity, completeness, and quality were ensured through internal and external revisions. 
Management and Follow-Up

All patients had a standard TUR with curative intent followed by a re-TUR at 4-6 weeks following the initial TUR [3]. Informed consent was obtained from each patient. Complete resection of all papillary tumors was a condition for BCG therapy in concordance with the EAU guidelines. Re-TUR generally included a fractioned resection of all visible lesions, depth resection of base and borders of previous resection area, and biopsy of any abnormal mucosal area. Re-TUR was usually performed by the same urologist who performed the first TUR [18]. Pathological evaluation was carried out according to the TNM system of Union for International Cancer Control and to the 1973 World Health Organization grading classification [19]. Patients with NMIBC on re-TUR and those with no residual tumor received an induction 6 weeks course of intravesical BCG followed by standard maintenance scheme, which consisted of intravesical BCG - standard dose - every week for 3 weeks given at $3,6,12,18,24,30$, and 36 months from initiation of therapy [20]. Only 303 (29\%) patients completed the treatment protocol as planned [21]. All patients were generally followed with cystoscopy and voiding urine cytology every 3-4 months for the first and second year, every 6 months for the third and fourth year, and annually thereafter. Diagnostic imaging of the upper tract was performed at least annually or when clinically indicated. Recurrence was defined as any tumor on follow-up and progression as MIBC on follow-up. Patients with muscle invasive disease on re-TUR and those who failed BCG underwent RC [3].

Endpoints were time to RFS, PFS, OS, and CSS. The cause of death was determined by the treating physician, based on chart review corroborated by death certificates when possible [22].

\section{Statistical Analysis}

We divided patients into 2 groups according to re-TUR results: pT0 or NMIBC other than T1 HG/G3 (Group A) and T1 HG/G3 (Group B) on re-TUR. Associations of T1 HG/G3 on re-TUR with categorical variables were assessed using $\chi^{2}$ tests; differences in continuous variables were analyzed using Mann-Whitney $U$ test. Univariable and multivariable logistic regression analyses were done to identify predictors of T1 HG/G3 on re-TUR. Kaplan-Meier method was used to estimate RFS, PFS, OS, and CSS; log-rank tests were applied for pair-wise comparison of survival. Univariable and multivariable Cox regression models addressed associations with RFS, PFS, OS, and CSS adjusting for the effects of standard clinicopathologic features. All $p$ values were 2 -sided, and statistical significance was defined as a $p<0.05$. Statistical analyses were performed using Stata 14.0 statistical software (Stata Corp., College Station, TX, USA).

\section{Results}

\section{Association of T1 HG/G3 on Re-TUR with Clinical} and Pathologic Characteristics

From a total of 1046 patients with T1 HG/G3 on the initial TUR 257 (24.6\%) had residual T1 HG/G3 on reTUR. Residual T1 HG/G3 was associated with the presence of concomitant carcinoma in situ (CIS), multiple and large tumors $(>3 \mathrm{~cm})$ on the initial TUR $(p \leq 0.001$; $p=0.01 ; p=0.03$, respectively). We did not find an association with age, gender, and smoking status (Table 1). On univariable analyses, current smoking status (OR $1.44, p=0.03$ ), tumor size (OR $1.38, p=0.03$ ), tumor multifocality (OR 1.44, $p=0.01$ ), and concomitant CIS (OR $2.05, p<0.001$ ) were all associated with T1 HG/G3 on reTUR. On multivariable analysis, tumor multifocality (OR $1.36, p=0.03$ ) and concomitant CIS (OR 2.01, $p=0.001$ ) remained significantly associated with $\mathrm{T} 1 \mathrm{HG} / \mathrm{G} 3$ on reTUR (Table 2).

\section{Association of T1 HG/G3 on re-TUR with Disease \\ Recurrence and Progression}

Within a median follow-up of 26 months, (interquartile range [IQR] 9-47), 203 (79\%) of the 257 patients with T1 HG/G3 on re-TUR experienced disease recurrence and within a median follow-up of 43 months, (IQR 36-58), 105 (40.9\%) disease progression compared with $475(60.2 \%)$ and $198(25.1 \%)$ patients with pT0 or NMIBC other than T1 HG/G3. On Kaplan-Meier analyses, residual T1 HG/G3 was associated with both worse RFS and PFS $(p<0.001)$. Five-year RFS and PFS were $17 \%$ (95\% CI $11.8-23)$ and $58.2 \%$ (CI 50.7-65) in patients with residual T1 HG/G3, compared to $36.7 \%$ (CI 32.8-40.6) and 71.4\% (CI 67.3-75.2) in patients with pT0 or NMIBC other than T1 HG/G3 (Fig. 1a, b). T1 HG/G3 at re-TUR was associated with worse RFS (hazard ratio [HR] 1.72, CI 1.46-2.03, $p<0.001)$ and PFS (HR 1.78, CI $1.4-2.26, p<0.001)$ on univariable Cox regression analyses. When adjusted for the effects of standard clinical and pathologic features from the initial TUR, residual T1 HG/G3 retained its significant association with both RFS (HR 1.63, CI 1.38-1.93, $p<0.001$ ) and PFS (HR 1.56, CI $1.22-1.98, p<0.001)$. Addition of this information from the re-TUR to a model that included the features of the initial TUR improved C-Index of the later by $4.1 \%$ for prediction disease recurrence and $2.3 \%$ for disease progression (Table 3).

\section{Association of T1 HG/G3 on re-TUR with Overall and CSS}

Within a median follow-up of 48 months (IQR 4068), 59 (22.9\%) of the 257 patients with T1 HG/G3 on re-TUR died with 32 (12.5\%) succumbing to BC compared with 91 (11.5\%) and $45(5.7 \%)$ patients with pT0 or NMIBC other than T1 HG/G3. On Kaplan-Meier analyses, residual T1 HG/G3 was associated with both worse OS and CSS $(p<0.001)$. Five-year OS and CSS were $73.7 \%$ (CI 66.3-79.7) and $84.5 \%$ (CI 77.8-89.3) in 
Table 1. Association from the features on initial T1 HG TURof bladder tumor specimen with residual T1 HG/ G3 or other NMIBC on re-TUR $(n=1,046)$ patients treated with maintenance BCG

\begin{tabular}{|c|c|c|c|c|}
\hline & All patients & Group A & Group B & $p$ value \\
\hline Total, $n(\%)$ & 1,046 & $789(75.4)$ & $257(24.6)$ & \\
\hline Age, years, mean (range) & $69.94(46-87)$ & 69.96 & 69.87 & 0.88 \\
\hline \multicolumn{5}{|l|}{ Gender, $n(\%)$} \\
\hline Male & $864(82.6)$ & $650(82.4)$ & $214(82.9)$ & \multirow[t]{2}{*}{0.75} \\
\hline Female & $182(17.4)$ & $139(17.6)$ & $43(17.1)$ & \\
\hline \multicolumn{5}{|l|}{ Smoking status, $n(\%)$} \\
\hline Never & $297(28.4)$ & $234(29.7)$ & $63(24.5)$ & \multirow[t]{3}{*}{0.052} \\
\hline Current & $485(46.4)$ & $249(44.2)$ & $136(52.9)$ & \\
\hline Former & $264(25.2)$ & $206(26.1)$ & $58(22.6)$ & \\
\hline \multicolumn{5}{|l|}{ Concomitant CIS, $n(\%)$} \\
\hline No & $896(85.7)$ & $695(88.1)$ & $201(78.2)$ & \multirow[t]{2}{*}{$<0.001$} \\
\hline Yes & $150(14.3)$ & $94(11.9)$ & $56(21.8)$ & \\
\hline \multicolumn{5}{|l|}{ Multifocality, $n(\%)$} \\
\hline Single & $585(55.9)$ & $459(58.1)$ & $126(49.9)$ & \multirow[t]{2}{*}{0.01} \\
\hline Multiple & $461(44.1)$ & $330(41.9)$ & $131(50.1)$ & \\
\hline \multicolumn{5}{|l|}{ Size, $\mathrm{cm}, n(\%)$} \\
\hline$<3$ & $371(35.5)$ & $294(37.3)$ & $77(30)$ & \multirow[t]{2}{*}{0.03} \\
\hline$\geq 3$ & $675(64.5)$ & $495(62.7)$ & $180(70)$ & \\
\hline
\end{tabular}

Group A: pT0 or NMIBC other than T1 HG/G3 on re-TUR, Group B: T1 HG/G3 on re-TUR.

TUR, transurethral resection; NMIBC, non-muscle invasive bladder cancer; BCG, Bacillus Calmette-Guérin; HG, high grade; CIS, carcinoma in situ.

Table 2. Univariable and multivariable logistic regression analyses predicting residual T1 HG/G3 disease on reTUR in 1,046 patients with primary T1 HG/G3

\begin{tabular}{|c|c|c|c|c|c|c|}
\hline \multirow[t]{3}{*}{ Variables } & \multicolumn{6}{|c|}{ T1 HG/G3 on re-TUR } \\
\hline & \multicolumn{3}{|c|}{ univariable } & \multicolumn{3}{|c|}{ multivariable } \\
\hline & OR & $95 \% \mathrm{CI}$ & $p$ value & OR & $95 \% \mathrm{CI}$ & $p$ value \\
\hline Age cont., years & 0.99 & $0.98-1.01$ & 0.89 & 1 & $0.98-1.01$ & 0.9 \\
\hline Gender, female vs. male & 0.93 & $0.64-1.36$ & 0.74 & 0.92 & $0.62-1.36$ & 0.69 \\
\hline Smoking status & & & Ref. & & & \\
\hline Current & 1.44 & $1.02-2.03$ & 0.03 & 1.34 & $0.95-1.91$ & 0.09 \\
\hline Former & 1.04 & $0.69-1.56$ & 0.82 & 0.96 & $0.63-1.45$ & 0.85 \\
\hline Multifocality, yes vs. no & 1.44 & $1.09-1.91$ & 0.01 & 1.36 & $1.02-1.81$ & 0.03 \\
\hline Size $(3 \mathrm{~cm}$ cut-off $)$ & 1.38 & $1.02-1.88$ & 0.03 & 1.34 & $0.98-1.82$ & 0.06 \\
\hline Concomitant CIS, yes vs. no & 2.05 & $1.42-2.97$ & $<0.001$ & 2.01 & $1.38-2.92$ & $<0.001$ \\
\hline
\end{tabular}

TUR, transurethral resection of bladder tumor; CIS, carcinoma in situ; HG, high grade.

patients with T1 HG/G3 on re-TUR compared to $89.8 \%$ (CI 86.6-92.3) and 95.7\% (CI 93.4-97.3) in patients with pT0 or NMIBC other than T1 HG/G3 (Fig. 2a, b). T1 HG/G3 on re-TUR was associated with OS (HR 1.93, CI $1.39-2.68, p<0.001$ ) and CSS (HR 2.11, CI 1.34-3.32, $p<$ 0.001 ) on univariable Cox regression analyses. When adjusted for the effects of clinical and pathologic features from the initial TUR, T1 HG/G3 on re-TUR retained its statistical significant association with both OS (HR 1.81, CI 1.29-2.54, $p=0.001$ ) and CSS (HR 1.82, CI 1.14-2.92, $p=0.01$ ). Addition of T1 HG/G3 on re-TUR to a base model including initial TUR information improved the C-Index of the later by $1.5 \%$ for OS and by $1.9 \%$ for CSS (Table 4).
10

Urol Int 2018;101:7-15

DOI: $10.1159 / 000490765$
Ferro et al. 
Fig. 1. Comparison of RFS (a) and PFS (b) between patients with T1 HG/G3 (Group $\mathrm{B})$ and those with $\mathrm{T} 0$ or non-muscle invasive BC other than T1 HG/G3 (Group A) on re-TUR in 1,046 patients with primary T1 HG/G3 treated with maintenance BCG. RFS, recurrence-free survival; PFS, progression-free survival; BC, bladder cancer; BCG, Bacillus Calmette Guerin.

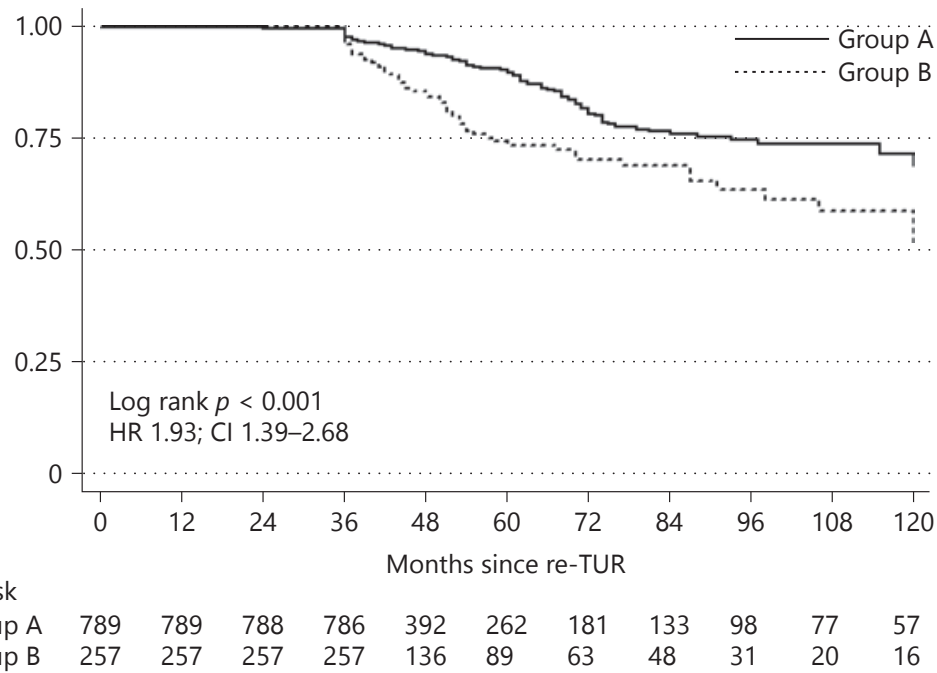

Number at risk

$\begin{array}{lllllllllllll} & \text { Group A } & 789 & 789 & 788 & 786 & 392 & 262 & 181 & 133 & 98 & 77 & 57 \\ \text { a } & \text { Group B } & 257 & 257 & 257 & 257 & 136 & 89 & 63 & 48 & 31 & 20 & 16\end{array}$

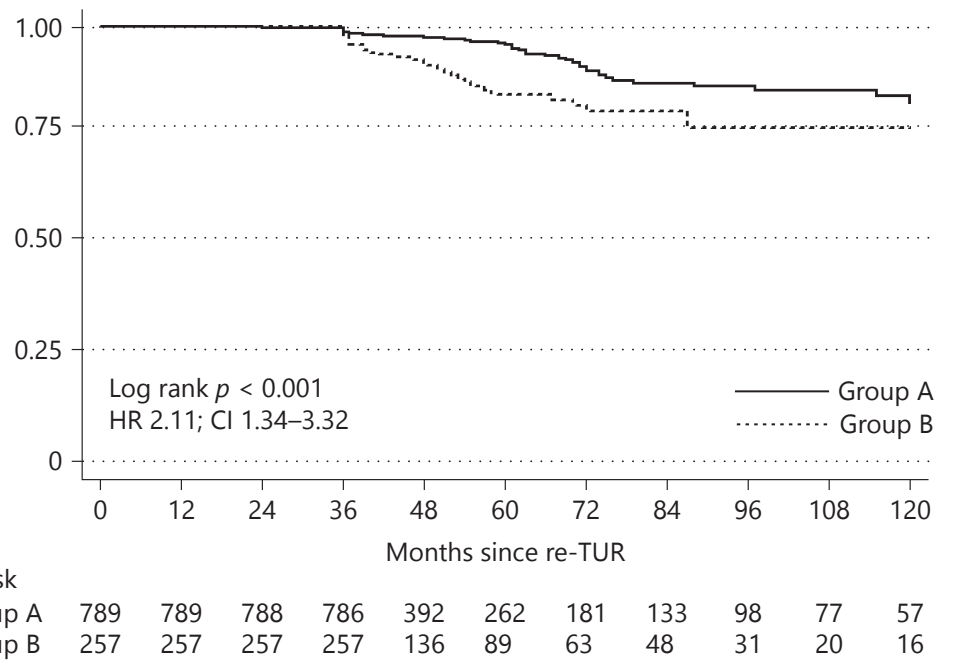

Number at risk

$\begin{array}{lllllllllllll} & \text { Group A } & 789 & 789 & 788 & 786 & 392 & 262 & 181 & 133 & 98 & 77 & 57 \\ \text { b } & \text { Group B } & 257 & 257 & 257 & 257 & 136 & 89 & 63 & 48 & 31 & 20 & 16\end{array}$

\section{Discussion}

We found that $24.6 \%$ of patients with initial T1 HG/G3 had residual T1 HG/G3. This is in agreement with the previous literature. For example, Herr et al. [16] reported that $23 \%$ patients with T1 G3 at initial TUR had residual T1 G3 on re-TUR. Similarly, Vasdev et al. [23] found that $23.8 \%$ patients with T1G3 on TUR had residual T1 G3 on reTUR. On the other hand, Gontero et al. [15] reported even a higher rate (30.9\%) in a multicenter international study. Recently, a prospective study that included 198 patients with T1 HG/G3 also showed that $1 / 4$ of patients have T1 HG/G3 on re-TUR; they also demonstrated that extent of T1 invasion did not eliminate the need for re-TUR [24].
In our study, T1 HG/G3 on re-TUR was associated with adverse pathological features such as multifocality, larger tumor size, and concomitant CIS. Of note, multifocality and the presence of concomitant CIS were both independent predictors of residual T1 HG/G3 on re-TUR. This is in accordance with reports from smaller studies in which both were found to be predictors for residual T1 HG/G3 $[12,25-28]$. While, it is important to identify patients who are likely to harbor residual T1 HG/G3 on re-TUR, this matters only if $\mathrm{T} 1$ on re-TUR impacts progression.

We found that residual T1 HG/G3 for T1 HG/G3 confers worse oncologic outcomes. Previous studies already reported higher recurrence (45\% [29] to $84 \%$ [30]) and progression rates (16\% [27] to $76 \%$ - includes 

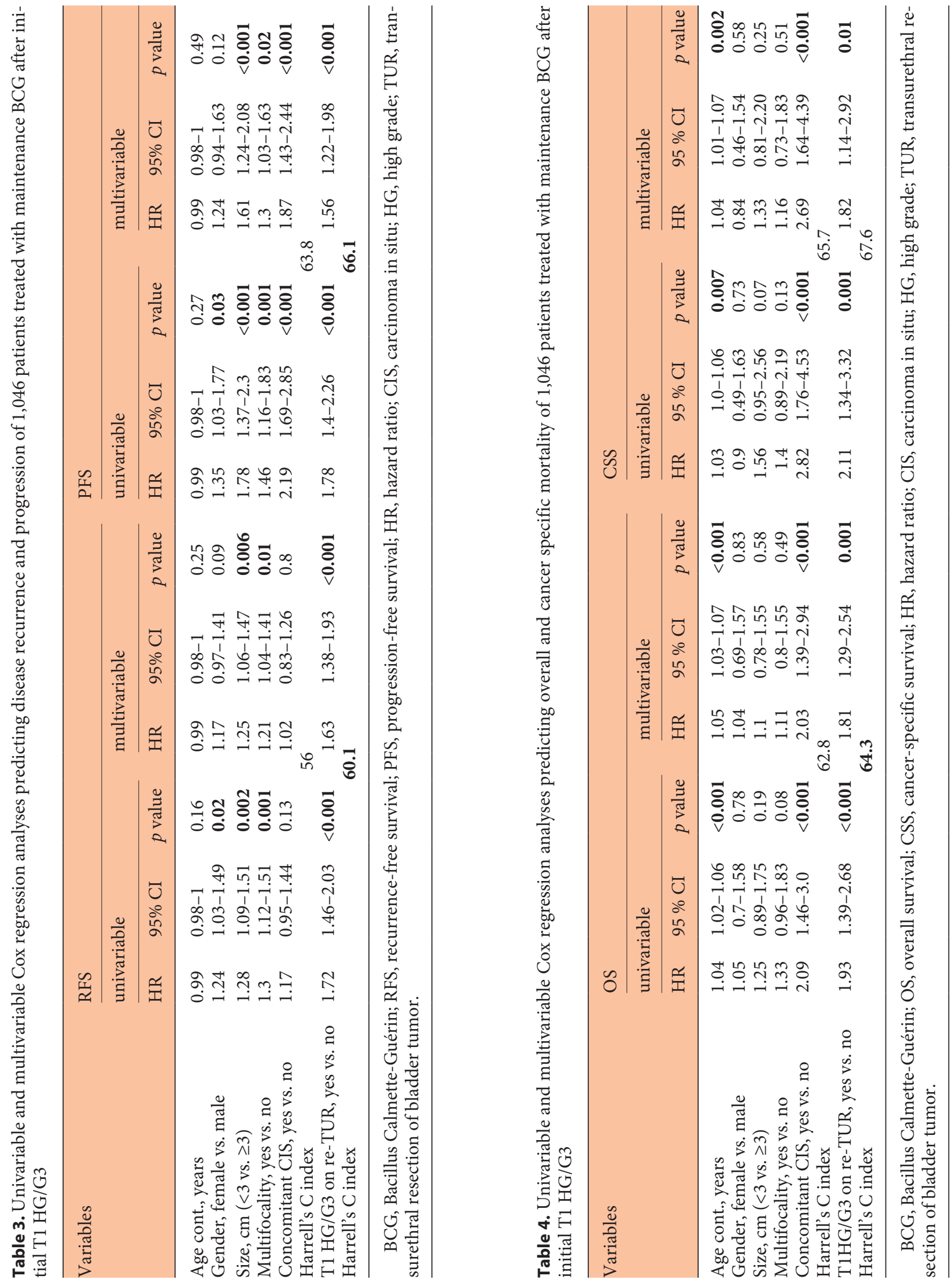
Fig. 2. Comparison of OS (a) and CSS (b) between patients with T1 HG/G3 (Group $\mathrm{B})$ and those with T0 or non-muscle invasive BC other than T1 HG/G3 (Group A) on re-TUR in 1,046 patients with primary T1 HG/G3 treated with maintenance BCG. OS, overall survival; CSS, cancer-specific survival; BC, bladder cancer; BCG, Bacillus Calmette Guerin.

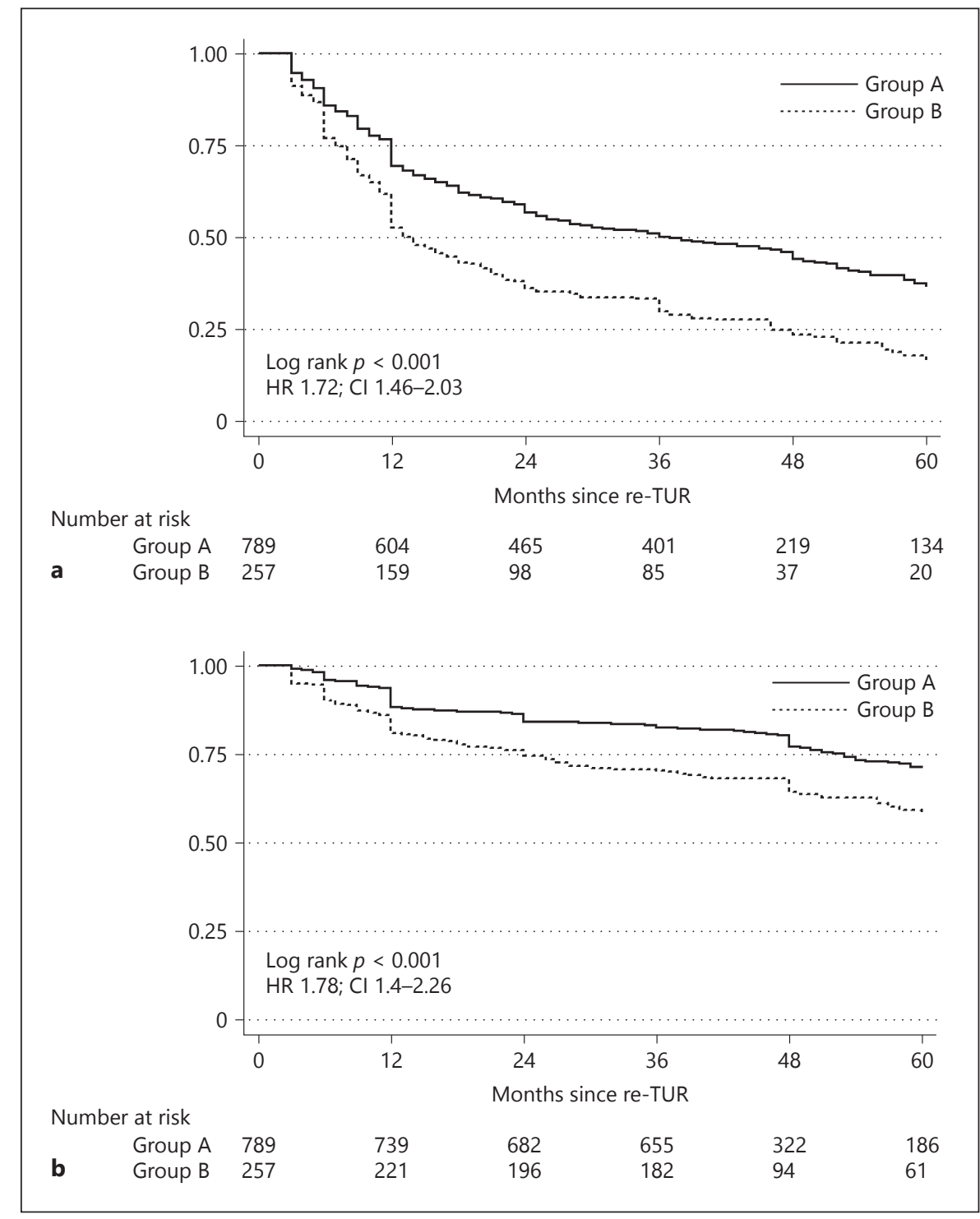

patients with stage and grade progression) [16] in patients with T1 HG/G3 on re-TUR compared to the other subgroups of NMIBC. Herr et al. [16] reported that $90 \%$ of T1G3 patients experienced disease recurrence and $76 \%$ had disease progression (mean follow-up of 78 months). These rates are lower than those in our study, probably compared due to longer follow-up and differences in the BCG schedule scheme as only 138 (24\%) patients received 2 or more than 2 BCG courses. In a prospective randomized study comparing BCG with epirubicin and interferon- $\alpha 2 \mathrm{~b}$ in patients with T1 residual tumor on re-TUR was significantly associated with an increased risk of recurrence as well as treatment failure [31]. Various mono-center studies confirmed that residual tumor on re-TUR is a harbinger for disease recurrence and progression compared to $\mathrm{pT} 0$ on re-TUR $[32,33]$. Disease progression of NMIBC is usually associated with worse survival compared to the novo MIBC [34].

We found that residual T1 HG/G3 on re-TUR for T1 HG/G3 increased the accuracy of standard models for predicting all 3 factors - disease recurrence, progression, and survival. While, residual T1 HG/G3 was associated with a $63 \%$ increased risk of disease recurrence and a $56 \%$ increased risk of disease progression, it also increased the risk of all-cause mortality by $81 \%$ and that of CSS by $82 \%$. Similarly, Kamiya et al. [27] showed, in their multicenter study, that T1 on re-TUR negatively impacted CSS in pa- 
tients with primary HG NMIBC. This information should be taken into consideration for the clinical decision regarding early $\mathrm{RC}$, as it has previously been shown that patients with de novo T2 have more favorable longterm disease-free survival compared to patients who experienced disease progression $[35,36]$. Indeed, many patients at time of early RC are upstaged (pT2 or greater, $41 \%)$ and $12.7 \%$ have even lymph node metastases [36, 37].

Despite this being the largest study to our knowledge investigating the prognostic value of residual T1 HG/G3 on re-TUR in patients with primary T1 HG/G3 treated with maintenance BCG, some limitations should be considered. First, its retrospective nature carries some intrinsic limitations. Second, some other factors could have been considered because they have been recognized as possible prognostic factors in T1 HG/G3 patients such as markers of systemic inflammation response [38, 39], lymphovascular invasion [40], and variant histology [41]. Third, due to the multi-center design, there is heterogeneity, as multiple surgeons and pathologists were implicated; however, in all centers, slides were reviewed by dedicated uro-pathologists, who reported that a complete first TUR and time for RC was only in case of progression to MIBC. While, with larger follow-up, the effect of T1 HG/G3 on re-TUR may change, its prognostic value would, however, remain the same.

\section{Conclusion}

Residual T1 HG/G3 tumor at re-TUR confers a statistically and prognostically worse prognosis in patients with primary T1 HG/G3 treated with maintenance BCG. Multifocality and concomitant CIS on primary TUR predict the risk of finding T1 HG/G3 on re-TUR. Patients with residual T1 HG/G3 for primary T1 HG/G3 are very likely to fail BCG therapy alone and should be considered for intensified therapy such as early RC or novel trials of immune checkpoint-inhibitors or device-assisted intravesical therapy.

\section{Acknowledgments}

M.D.V is supported by the Scholarship Foundation of the Republic of Austria - OeAD and by the EUSP Scholarship - EAU.

\section{Ethical Standards}

This study has been approved by the appropriate Ethics Committee.

\section{Disclosure Statement}

The authors declare that they have no conflicts of interest to disclose.

\section{Author Contribution}

Protocol/project development: M.F., M.D.V., V.M., and S.F.S. Data collection or management: M.D.V., M.F., F.C., G.L., S.M.D.S., R.H., G.G., G.M.B., E.D.B., R.D., S.P., M.B., R.S., G.L.A., P.B., E.L., G.G., R.A., N.C., A.R.A.F., P.V., M.B., V.S., G.I.R., G.M., O.C., V.M., and S.F.S. Data analysis: M.D.V., M.F., F.C., G.L., S.M.D.S., R.H., G.G., G.M.B., E.D.B., R.D., S.P., M.B., R.S., G.L.A., P.B., E.L., G.G., R.A., N.C., A.R.A.F., P.V., M.B.,V.S., G.I.R., G.M., O.C., V.M., and S.F.S. Manuscript writing/editing: M.D.V., M.F., F.C., G.L., S.M.D.S., R.H., G.G., G.M.B., E.D.B., R.D., S.P., M.B., R.S., G.L.A., P.B., E.L., G.G., R.A., N.C., A.R.A.F., P.V., M.B.,V.S., G.I.R., G.M., O.C., V.M., and S.F.S.

\section{References}

1 Torre LA, Bray F, Siegel RL, Ferlay J, LortetTieulent J, Jemal A: Global cancer statistics, 2012. CA Cancer J Clin 2015;65:87-108.

2 Kamat AM, Hegarty PK, Gee JR, Clark PE, Svatek RS, Hegarty N, et al: ICUD-EAU international consultation on bladder cancer 2012: screening, diagnosis, and molecular markers. Eur Urol 2013;63:4-15.

3 Babjuk M, Böhle A, Burger M, Capoun O, Cohen D, Compérat EM, et al: EAU Guidelines on Non-Muscle-invasive Urothelial Carcinoma of the Bladder: Update 2016. Eur Urol 2017;71:447-461.

4 Martin-Doyle W, Leow JJ, Orsola A, Chang SL, Bellmunt J: Improving selection criteria for early cystectomy in high-grade 11 bladder cancer: a meta-analysis of 15,215 patients. J Clin Oncol 2015;33:643-650.

5 Chang SS, Boorjian SA, Chou R, Clark PE, Daneshmand S, Konety BR, et al: Diagnosis and treatment of non-muscle invasive bladder cancer: AUA/SUO Guideline. J Urol 2016; 196:1021-1029.

6 Poletajew S, Zapała P, Radziszewski P: Safety and efficacy of intravesical bacillus calmette-guérin immunotherapy in patients with non-muscle-invasive bladder cancer presenting with asymptomatic bacteriuria: a systematic review. Urol Int 2017;99: $1-5$.
7 Steinberg RL, Thomas LJ, O'Donnell MA: Bacillus calmette-guérin (BCG) treatment failures in non-muscle invasive bladder cancer: what truly constitutes unresponsive disease. Bladder Cancer 2015;1:105116.

8 Daneshmand S: Determining the role of cystectomy for high-grade $\mathrm{T} 1$ urothelial carcinoma. Urol Clin North Am 2013;40:233-247.

9 Badalato GM, Gaya JM, Hruby G, Patel T, Kates M, Sadeghi N, et al: Immediate radical cystectomy vs conservative management for high grade cT1 bladder cancer: is there a survival difference? BJU Int 2012;110:14711477. 
10 Shariat SF, Ashfaq R, Karakiewicz PI, Saeedi O, Sagalowsky AI, Lotan Y: Survivin expression is associated with bladder cancer presence, stage, progression, and mortality. Cancer 2007;109:1106-1113.

11 Byrne RR, Shariat SF, Brown R, Kattan MW, Morton RA JR, Wheeler TM, et al: E-cadherin immunostaining of bladder transitional cell carcinoma, carcinoma in situ and lymph node metastases with long-term followup. J Urol 2001;165:1473-1479.

12 Fritsche HM, Burger M, Svatek RS, Jeldres C, Karakiewicz PI, Novara G, et al: Characteristics and outcomes of patients with clinical T1 grade 3 urothelial carcinoma treated with radical cystectomy: results from an international cohort. Eur Urol 2010;57:300-309.

13 Breyer J, Otto W, Wirtz RM, Wullich B, Keck B, Erben P, et al: ERBB2 expression as potential risk-stratification for early cystectomy in patients with pT1 bladder cancer and concomitant carcinoma in situ. Urol Int 2017;98: 282-289.

14 Woldu SL, Bagrodia A, Lotan Y: Guideline of guidelines: non-muscle-invasive bladder cancer. BJU Int 2017;119:371-380.

15 Gontero P, Sylvester R, Pisano F, Joniau S, Oderda M, Serretta V, et al: The impact of retransurethral resection on clinical outcomes in a large multicentre cohort of patients with T1 high-grade/Grade 3 bladder cancer treated with bacille Calmette-Guérin. BJU Int 2016; 118:44-52.

16 Herr HW, Donat SM: A re-staging transurethral resection predicts early progression of superficial bladder cancer. BJU Int 2006;97: 1194-1198.

17 Divrik RT, Sahin AF, Yildirim U, Altok M, Zorlu F: Impact of routine second transurethral resection on the long-term outcome of patients with newly diagnosed pT1 urothelial carcinoma with respect to recurrence, progression rate, and disease-specific survival: a prospective randomised clinical trial. Eur Urol 2010;58:185-190.

18 Del Zingaro M, Bruno R, Nunzi E, Porena M, Mearini L: First and second transurethral resections in intermediate-high risk bladder cancer: impact of the surgeon's volume on the recurrence and progression of primary bladder cancer. Minerva Urol Nefrol 2016;68: 194-203.

19 Balan D, Martha O, Chibelean CB, Tataru S, Voidezan S, Sin A, et al.: Comparison of 10 year overall survival between patients with G1 and G2 grade Ta bladder tumors. Medicine (Baltimore) 2018;97:e0522.

20 Hofbauer SL, Shariat SF, Chade DC, Sarkis AS, Ribeiro-Filho LA, Nahas WC, et al: The moreau strain of Bacillus Calmette-Guerin (BCG) for high-risk non-muscle invasive bladder cancer: an alternative during worldwide BCG shortage? Urol Int 2016;96:4650.

21 Lamm DL: Efficacy and safety of bacille Calmette-Guérin immunotherapy in superficial bladder cancer. Clin Infect Dis 2000; 31(suppl 3):S86-S90.

22 Rink M, Fajkovic H, Cha EK, Gupta A, Karakiewicz PI, Chun FK, et al: Death certificates are valid for the determination of cause of death in patients with upper and lower tract urothelial carcinoma. Eur Urol 2012;61:854-855.

23 Vasdev N, Dominguez-Escrig J, Paez E, Johnson MI, Durkan GC, Thorpe AC: The impact of early re-resection in patients with $\mathrm{pT} 1$ high-grade non-muscle invasive bladder cancer. Ecancermedicalscience 2012;6:269.

24 Audenet F, Retinger C, Chien C, Benfante NE, Bochner $\mathrm{BH}$, Donat SM, et al: Is restaging transurethral resection necessary in patients with non-muscle invasive bladder cancer and limited lamina propria invasion? Urol Oncol 2017;35:603.e1-e603.e5.

25 Takaoka E, Matsui Y, Inoue T, Miyazaki J, Nakashima M, Kimura T, et al: Risk factors for intravesical recurrence in patients with high-grade T1 bladder cancer in the second TUR era. Jpn J Clin Oncol 2013;43:404-409.

26 Gill TS, Das RK, Basu S, Dey RK, Mitra S: Predictive factors for residual tumor and tumor upstaging on relook transurethral resection of bladder tumor in non-muscle invasive bladder cancer. Urol Ann 2014;6:305-308.

27 Kamiya N, Suzuki H, Suyama T, Kobayashi M, Fukasawa S, Sekita N, et al: Clinical outcomes of second transurethral resection in non-muscle invasive high-grade bladder cancer: a retrospective, multi-institutional, collaborative study. Int J Clin Oncol 2017;22: 353-358.

28 Turk H, Un S, Isoglu CS, Karabicak M, Ergani B, Yoldas M, et al: Factors that predict residual tumors in re-TUR patients. Afr J Urol 2016;22:67-70.

29 Holmang S: High-grade non-muscle-invasive bladder cancer: Is re-resection necessary in all patients before intravesical bacillus CalmetteGuerin treatment? Scand J Urol 2013;47:363369.

30 Bishr M, Lattouf JB, Latour M, Saad F: Tumour stage on re-staging transurethral resection predicts recurrence and progression-free survival of patients with high-risk non-muscle invasive bladder cancer. Can Urol Assoc J 2014;8:E306-E310.

31 Hemdan T, Johansson R, Jahnson S, Hellstrom P, Tasdemir I, Malmstrom PU: 5-year outcome of a randomized study comparing bacillus Calmette-Guerin with epirubicin and interferon- $\alpha 2 \mathrm{~b}$ in patients with $\mathrm{T} 1$ bladder cancer. J Urol 2014;191:1244-1249.

32 Sanseverino R, Napodano G, Campitelli A, Addesso M: Prognostic impact of ReTURB in high grade T1 primary bladder cancer. Arch Ital Urol Androl 2016;88:81-85.

33 Tae BS, Jeong CW, Kwak C, Kim HH, Moon $\mathrm{KC}, \mathrm{Ku} \mathrm{JH}$ : Pathology in repeated transurethral resection of a bladder tumor as a risk factor for prognosis of high-risk non-muscle-invasive bladder cancer. PLoS One 2017;12: e0189354.

34 Breau RH, Karnes RJ, Farmer SA, Thapa P, Cagiannos I, Morash C, et al: Progression to detrusor muscle invasion during urothelial carcinoma surveillance is associated with poor prognosis. BJU Int 2014;113:900-906.

35 Vartolomei MD, Kiss B, Vidal A, Burkhard F, Thalmann GN, Roth B: Long-term results of a prospective randomized trial assessing the impact of re-adaptation of the dorsolateral peritoneal layer after extended pelvic lymph node dissection and cystectomy. BJU Int 2016;117:618-628.

36 Gupta A, Lotan Y, Bastian PJ, Palapattu GS, Karakiewicz PI, Raj GV, et al: Outcomes of patients with clinical $\mathrm{T} 1$ grade 3 urothelial cell bladder carcinoma treated with radical cystectomy. Urology 2008;71:302-307.

37 Karakiewicz PI, Shariat SF, Palapattu GS, Perrotte P, Lotan Y, Rogers CG, et al: Precystectomy nomogram for prediction of advanced bladder cancer stage. Eur Urol 2006;50: 1254-1260; discussion 1261-1262.

38 Martha O, Porav-Hodade D, Bălan D, Tătaru OS, Sin A, Chibelean CB, et al: Easily available blood test neutrophil-to-lymphocyte ratio predicts progression in high-risk non-muscle invasive bladder cancer. Rev Romana Med Lab 2017;25:181-189.

39 Mbeutcha A, Shariat SF, Rieken M, Rink M, Xylinas E, Seitz C, et al: Prognostic significance of markers of systemic inflammatory response in patients with non-muscle-invasive bladder cancer. Urol Oncol 2016;34:483. e17-e483.e24.

40 Shariat SF, Svatek RS, Tilki D, Skinner E, Karakiewicz PI, Capitanio U, et al: International validation of the prognostic value of lymphovascular invasion in patients treated with radical cystectomy. BJU Int 2010;105: 1402-1412.

41 Moschini M, D’Andrea D, Korn S, Irmak Y, Soria F, Compérat E, et al: Characteristics and clinical significance of histological variants of bladder cancer. Nat Rev Urol 2017;14:651668
HG T1 on Re-TUR after Initial HG T1

Confers Worse Oncological Outcomes
Urol Int 2018;101:7-15

DOI: $10.1159 / 000490765$ 